Culture et histoire dans l'espace roman

4 | 2010

Les représentations du corps dans la litterature latinoaméricaine

\title{
Les corps malmenés de Sergio Ramírez
}

Images d'un Nicaragua meurtri

\section{Nathalie Besse}

\section{(2) OpenEdition}

Journals

Édition électronique

URL : https://journals.openedition.org/cher/8449

DOI : 10.4000/cher.8449

ISSN : 2803-5992

Éditeur

Presses universitaires de Strasbourg

Édition imprimée

Date de publication : 30 juin 2010

Pagination : 103-116

ISBN : 978-2-35410-007-0

ISSN : 1968-035X

\section{Référence électronique}

Nathalie Besse, "Les corps malmenés de Sergio Ramírez », reCHERches [En ligne], 4 | 2010, mis en ligne le 15 décembre 2021, consulté le 24 janvier 2022. URL : http://journals.openedition.org/cher/ 8449 ; DOI : https://doi.org/10.4000/cher.8449

\section{cc) (1) (ㅇ)}

Ce(tte) œuvre est mise à disposition selon les termes de la Licence Creative Commons Attribution -

Pas d'Utilisation Commerciale - Partage dans les Mêmes Conditions 4.0 International. 


\title{
Les corps malmenés de Sergio Ramírez Images d'un Nicaragua meurtri
}

\author{
NATHALIE BESSE \\ Université de Strasbourg
}

\begin{abstract}
ans les romans de Sergio Ramírez, auteur incontournable des lettres nicaraguayennes, il n'y a guère de personnages dont le corps ne soit victime d'un humour féroce: grotesque, monstrueuse, mutilée, cette corporéité malmenée sert bien souvent en filigrane la dénonciation du malheur et de l'infamie qui meurtrissent son pays. Notre étude se concentrera sur: Margarita, está linda la mar (prix Alfaguara 1998), Sombras nada más (2002) et Mil y una muertes (2004) qui disent implicitement, par le corps des personnages, l'Histoire de la patrie.

Nous verrons comment le corps, objet de dérision, démythifié, voire diabolisé dans le cas du dirigeant, signifie l'identité abîmée, sinon avortée, de tout un pays. Du corps amputé à la mort des mythes nationaux, ou du corps souillé à une malédiction de la corruption, c'est de mort que nous parlent les corps aberrants de ces fictions. Et sans doute des illusions perdues de Sergio Ramírez.
\end{abstract}

\section{Le corps grotesque: entre dérision et dénonciation}

Les romans de Sergio Ramírez abondent en corps disgracieux et difformes, tantôt avilis par quelque précision physiologique rebutante, tantôt abîmés par la maladie, sciemment enlaidis par les soins goguenards du romancier. Parfois outrancièrement moqués. Même sous leur forme la plus atténuée, ces estocades portées au corps dépeignent à tout le moins des personnages amoindris. 
Ainsi en est-il du protagoniste de Sombras nada más, Alirio Martinica (l'ancien bras droit du dictateur Somoza Debayle) que les sandinistes capturent peu de temps avant le triomphe révolutionnaire et qui doit répondre de ses actes devant un tribunal populaire. Ce personnage falot et soumis par les futurs vainqueurs donne à lire par le corps l'anéantissement de la personne: dépersonnifié par le substantif «el reo» durant quelque trente-cinq pages, attaché, enfermé, et donc privé de sa liberté, on le voit agenouillé dans l'obscurité, tête basse ou larmoyant. Puis, lorsqu'il paraît devant le peuple, c'est par le corps encore que sa maîtresse achève de lui ôter toute dignité en le taxant d'impuissant et de pédophile, avant qu'on ne le mène au mur des fusillés en caleçons et dans la cage où se trouvait auparavant son singe.

Cette dévirilisation qui contient une castration symbolique, et cette animalisation qui remet en cause l'humanité même du personnage, humilient corps et être, indissociablement liés. On ne saurait en effet tourmenter ce corps porteur de l'être de chacun, sans ébranler ce dernier dans son identité même. Mépriser le corps, c'est porter atteinte à la personne; offenser la personne, c'est aussi, bien souvent, inscrire dans le corps certaines blessures. Car sans se réduire à la corporalité, l'existence est corporelle. Sans le corps, que serions-nous? puisque pour reprendre la formule bien connue de Freud dans Le Moi et le Ça: «le Moi est avant tout une entité corporelle» (1979: 194). À quoi s'ajoute l'enseignement de Durkheim selon lequel le corps s'avère un "facteur d'individuation» (1968: 386), ce qui permet donc le passage du corps-objet vers le corps-sujet. Nous verrons que chez Sergio Ramírez, c'est un mouvement inverse qui paraît se produire, comme une involution de la personne ne devenant plus qu'un corps, semble-t-il dépossédé de son âme.

L'exemple d'Alirio Martinica n'est pourtant pas le plus représentatif des corps maltraités de Sergio Ramírez sur un point: l'adversité dans laquelle il se trouve ne prête guère à rire alors que le plus souvent, c'est la veine burlesque qui régit les descriptions corporelles de personnages face à leur propre mort, parfois agonisants. Dans Mil y una muertes, un cortège de décès présentés avec une certaine frivolité, dévaste la famille aristocratique de l'Archiduc Luis Salvador, bienfaiteur des Baléares: pour n'en citer que quelques-uns, la princesse Mathilde agonise sur un lit hydrostatique suite aux brûlures provoquées par sa robe prenant feu; une cousine périt dans les flammes d'un bazar appelé La Charité; une autre est assassinée à Genève par un anarchiste fanatique; un cousin meurt en tombant d'un cheval devenu 
fou; jusqu'au secrétaire de l'Archiduc qui succombe à une insolation après avoir attendu trop longtemps en plein soleil sa bien-aimée.

Autant de disparitions cocasses, traitées avec la plus totale légèreté, comme si dans la grande tragicomédie de l'existence, il était salutaire de se rire du drame humain. Ce corps que la mort emportera, se prête aisément à cette dérision. Déjà dans Un Baile de máscaras (1995), le romancier mettait en scène cinq morts comiques autour de sa propre naissance; déjà dans Castigo Divino (1988), il rappelait, à grand renfort de médecin-légiste, d'autopsie et de viscères, que sans se réduire à l'anatomie, le corps est aussi, et peut-être d'abord, cette machine dont parlait Descartes, un corps dans lequel il n'est pas toujours aisé d'être comme «un pilote en son navire», et qui nous possède parfois plus que nous ne le possédons.

L'auteur récidive en l'espèce ainsi qu'en témoignent, dans Mil y una muertes, certaines affections qui n'épargnent pas les plus illustres artistes: un Flaubert chauve et édenté en raison du traitement qu'il doit prendre contre la syphilis, un Tourgueniev qui souffre au point de tenter de s'étrangler avec le fil du télégraphe, un Chopin tuberculeux materné par une George Sand virago. Sergio Ramírez a certes pris ce pli, depuis plusieurs fictions, de répondre au talent par la dégradation ou de se jouer de toute respectabilité - il n'est que d'évoquer le traitement des hommes de pouvoir bien souvent ridiculisés par le scatologique -; mais nul doute qu'il faille également s'interroger ici sur une mise à distance de la douleur et de la mort grâce à cette «anesthésie momentanée du cœur» (Bergson 1993:4) que serait le rire si l'on en croit le philosophe.

Rubén Darío lui-même, mythe nicaraguayen s'il en est, subit la raillerie de Sergio Ramírez. Le "Gran Panida» d'ailleurs "diminué" en une petite statue, est en effet dépeint dans Margarita, está linda la mar et derechef dans Mil y una muertes, en poète aviné, de ce fait boursouflé. La dégradation, et l'aliénation, du corps proviennent ici d'une dipsomanie qui émanerait à son tour de la neurasthénie du personnage: mens sana in corpore sano disait Juvénal, et corps malsain quand l'esprit lui-même est corrompu. Cet amant des Muses en outre ventru et peut-être rendu impuissant par ces intempérances, n'est pas sans rappeler les observations de Mikhail Bakhtine concernant le "bas" corporel associé aux sphères sexuelle et digestive. Celuici explique qu'après le ventre et les organes génitaux, ce sont la bouche et le séant, rappelant les besoins naturels, qui jouent le rôle le plus important dans le «réalisme grotesque» corporel (Bakhtine 1985: 315). 
Et en matière d'organisme grossier, c'est indéniablement le dictateur - Somoza García dans Margarita, está linda la mar, puis son fils Somoza Debayle dans Sombras nada más - qui s'avère brocardé de la pire manière qui soit au moyen du thème excrémentiel. En effet, dans le premier roman, Somoza, qui fut autrefois inspecteur de sanitaires, «caga por la barriga, [...] por medio de una válvula de goma" depuis la suppression du rectum et la greffe d'un anus artificiel sur le ventre (Ramírez 1998: 37). Et dans le deuxième ouvrage mentionné, le tyran est victime d'incontinence fécale dans la piscine où l'entourent ses collaborateurs qui n'osent fuir (Ramírez 2002: 370).

Mais au-delà de l'humiliation infligée au despote non sans hasard relié à l'abject, c'est le sens allégorique donné à cette grotesquisation accablante qui nous intéresse: Margarita, está linda la mar présente en effet Somoza comme un "gángster que sin tener culo se ha cagado en todo Nicaragua" (Ramírez 1998: 36), sur quoi renchérit Sombras nada más qui fait de l'anecdote truculente de la piscine une autre métaphore nationale: " ¡El somocismo no es más que pura mierda, y en esa mierda se bañan los serviles!»(Ramírez 2002: 394). Ainsi donc, l'impur dirigeant dont la corruption proverbiale n'est plus à prouver, condamne-t-il à la souillure son malheureux pays. Non moins qu'à l'Enfer, comme en témoigne cette assertion mêlant le scatologique au satanique, et ajoutant au corps grotesque le corps monstrueux : "El aire huele a azufre, a mierda de diablo» (Ramírez 1998: 263).

\section{Le corps monstrueux: la Bête et les «surhommes», emblèmes identitaires}

La diabolisation de Somoza n'est qu'un exemple parmi tant d'autres de caricature politique qui convertit volontiers le potentat en monstre. Ce dernier constitue, ainsi que le pensait Aristote, un être défectueux: «la nature ne s'y trouve pas réalisée adéquatement. Les monstres biologiques sont l'inadéquat, l'imparfait», explique Gilbert Lascault (2004: 248). Or, cet écart par rapport à la norme, ce corps "extrême", est perçu comme une menace contre toute forme d'existence, le monstre représentant une force qui s'oppose à celles qui constituent la vie. Non sans raison est qualifié de monstre celui qui, comme en proie aux pulsions de mort, abuse jusqu'à l'inconcevable de la cruauté et de la destruction. À cet égard, l'analogie entre le monstre et le tyran n'a rien de très surprenant, celui-ci laissant s'exprimer plus que d'autres la bête en lui dans sa dimension la plus effrayante.

C'est bien ce que signifie du reste la monstruosité de Somoza, incarnation même du Mal à en juger par les images apocalyptiques qui l'accompagnent: 
«Vergüenza para nosotros y para la armonía del universo, que la bestia asiente su pezuña en estas calles. [...] Ha nacido el anticristo, hermano. Y para nuestra desgracia, en Nicaragua", déplore un des conspirateurs (Ramírez 1998: 217). Car c'est à nouveau la perspective nationale qui intéresse le romancier. Et l'on voit bien comment, au-delà de la farce, une veine satirique à visée axiologique nous permet de ne pas oublier que ce monstre qu'est le dictateur, introduit sur sa terre le chaos et les ténèbres, comme un retour à l'informel, à l'incréé, autant dire au néant. Si donc "l'imagerie démonique qui greffe le bizarre et le risible sur le macabre et l'abject, se rattache à la catégorie esthétique du grotesque» (Duval et Martinez 2000 : 210), elle participe également d'une stratégie de la dénonciation tendant à rappeler "l'enfer" de la dictature qui voue à la mort une nation entière.

Le corps de l'oppresseur suffit à l'illustrer, et à montrer combien le corps est porteur de valeurs symboliques, combien il constitue à lui seul un langage - indépendamment de ses relations discrètes mais non moins essentielles avec l'inconscient et la société. Ici, la corporéité contient nombre d'archétypes, d'images cryptées dont on dénoue spontanément le sens, tant il est vrai que le corps constitue un formidable support pour transmettre certains messages. Idéel autant que charnel, le corps est aussi à lire, texte en soi. Donnant donc à penser. Et en l'occurrence, texte redresseur de torts, précisément dénoncés via le corps.

Pour autant, les exemples monstrueux de Margarita, está linda la mar ne sauraient se limiter aux personnages que nous qualifierons par commodité de "négatifs": il en est un autre en effet, incarnant plutôt le naïf quant à lui, affublé de sabots et explicitement animalisé puisqu'il s'agit de Quirón, inspiré, cela va de soi, du centaure éponyme, protégé de Rubén Darío dans le roman où celui-ci lui transmet, lors d'un acte télépathique, l'inestimable numen. Ce clin d'œil à un célèbre poème de Rubén Darío «El Coloquio de los Centauros» de Prosas Profanas, met en scène un personnage bienveillant aussi bien, Chiron est-il dans la mythologie le seul centaure bienfaisant, maître d'Esculape, dieu de la médecine.

«Plus souvent qu'on ne l'imaginerait, les formes monstrueuses peuvent être positivement valorisées", explique Gilbert Lascault (2004: 293), et ce Quirón qui, à deux reprises, nous est dépeint s'enfuyant au galop, «sus rudos cascos golpeando contra el empedrado, los lomos sudorosos» (Ramírez 1998: 278 ou 369), préserve par ailleurs la mémoire de ces emblèmes identitaires 
que seraient le poète Darío et Rigoberto López Pérez qui, tel le digne héritier de Sandino, assassinera Somoza.

Et s'agissant à nouveau de problématique nationale, Quirón va être outrageusement meurtri dans son corps par des marines qu'il a dénoncés pour profanation d'un cimetière où ils ont laissé libre cours à leur luxure, et qui vont donc perpétrer un acte d'une sauvagerie guère différente de celle communément pratiquée par Somoza et ses sbires; or, on sait la présence et le rôle délétères des États-Unis au Nicaragua, de fait animalisés et diabolisés au même titre que le tyran affidé dont ils ont grandement favorisé l'émergence au pouvoir: «El caos es el que siembran esos hijos del averno. [...] ¡Gorilas colorados, como los ha llamado el mismo Rubén!» (236).

Ce même Rubén n'échappe d'ailleurs pas à une certaine forme de monstruosité si l'on considère la disproportion de sa tête, elle-même due à un prodigieux encéphale. Ce cerveau, «[El] más portentoso de todos los siglos", pèserait, selon le docteur Debayle, quelque 1850 grammes - quand la moyenne pour un adulte est de 1375 - et parait d'autant plus indétrônable qu'il excède même celui d'un Victor Hugo (281 et 273). La tératologie montre que les monstres aux organes excessivement développés sont relativement nombreux, et que bien souvent une tête énorme domine un corps minuscule, ce qui confère un aspect plus drôle que véritablement inquiétant (Lascault 2004: 132). Ici, l'hypertrophie cérébrale du grand poète met en relief l'intelligence et le talent de ce "mythe" national, fierté de sa patrie.

Il en va de même de Sandino, paradigme de l'identité nicaraguayenne à l'instar de Rubén Darío selon l'auteur lui-même (Ramírez Una vida... : 2004, 198), et lui aussi doté d'un organe surdimensionné, "monstrueusement" membré pour ainsi dire: "unos testículos descomunales [...] enormes y sonrosados como la postura del ave fénix» (Ramírez 1998: 218). Le corps traduit ici une valeur morale puisque le sexe hyperbolique du héros nationaliste symbolise de toute évidence une irréductible vaillance, d'autant plus louable en terre nicaraguayenne que si l'on en croit l'un des conjurés qui blâme l'absence de courage de ses compatriotes, "éste es un país de eunucos» (218). Dans un jeu de métaphores corporelles symétriques d'ailleurs bien connues, si le priapisme définit la bravoure, l'absence de phallus souligne quant à elle la pleutrerie; mais cette image de la mutilation associée à tout un pays, va trouver un écho terrible dans la castration du brave. Car la monstruosité, c'est aussi celle pratiquée par la Bête elle-même ou par ses tortionnaires. 


\section{Le corps mutilé: un Nicaragua meurtri inhumant ses mythes}

Dans ce Nicaragua qui nous est présenté par les comploteurs antisomocistes de Margarita, está linda la mar, comme un pays de lâches, même le Juste qui, dans une parodie du mythe, fait renaître des cendres la virilité "fabuleuse" du rebelle Sandino - "que los huevos del ave fénix resurjan de las cenizas» (Ramírez 1998: 219) - sera atrocement mutilé puisque victime d'une émasculation post mortem. Cette barbarie provient d'une plaisanterie macabre du despote reprenant connaissance après l'attentat, et que son colonel prendra à la lettre: "ahora por lo menos... que le corten... los huevos [...]. Es para que... me le den... sopa de huevos... a la guardia...»; certains des autres conspirateurs auront à subir le même sort de leur vivant (348 et 373), entre autres exemples de violences faites au corps sous la dictature, que les romans de Sergio Ramírez, à fort ancrage historique pour la plupart, n'omettent pas de mentionner, sans pour autant s'y complaire.

Les monstres renvoient à de grandes peurs humaines, parmi lesquelles l'angoisse de la mutilation (Anne Carol in Le «monstre» humain 2005: 5). Et la perte d'organes, le démembrement, les tortures, sont autant de figures de l'“impouvoir" selon Gilbert Lascault (2004: 373). Or, le phallus, qui ne désigne pas en psychanalyse qu'une réalité anatomique mais contient une dimension symbolique, signifie, si l'on veut simplifier, la puissance. Si donc le petit pays est peuplé de "castrats", il paraît de surcroît contraint à l'impuissance quand bien même il essaierait de se libérer du monstre. Il est vrai que Somoza García finira par rendre l'âme, mais ses fils, de même engeance maudite, assureront la relève.

$\mathrm{Au}$ demeurant, l'excipit du roman ne cède-t-il en rien à l'optimisme puisqu'il décrit Quirón dérobant aux autorités le flacon de formol qui contient le sexe de Rigoberto pour l'emmener - comme il le fit des années auparavant avec l'urne où reposait le cerveau de Rubén Darío - «hacia el prostíbulo desierto, hacia la fuente de noche y de olvido, hacia la nada» (Ramírez 1998: 369). Les trois derniers substantifs mortuaires du roman peuvent être mis en parallèle avec le sort que connaît l'encéphale du poète d'abord caché dans un bordel tenu par un séide de Somoza, "La Caimana», puis dans un cimetière où l'on enterrait autrefois les foetus de grossesses honteuses. Et à nouveau la mort. Ces deux organes arrachés à leur corps et flottant dans leur récipient comme deux méduses, ne disent-ils pas précisément la mort des mythes nationaux nicaraguayens? Réduits à néant. Et avec eux, une identité mutilée ou avortée? Et faut-il entendre que le petit pays est définitivement condamné à la perte de créativité et de courage? 
L'exemple de Rubén Darío tend à corroborer cette analyse car lui aussi va subir une forme de mutilation, métaphore probable d'une atteinte à l'identité nationale. En effet, dans ce roman qui discrédite, outre le pouvoir politique, l'autorité scientifique, le poète doit être opéré par le docteur Debayle, un prétendu savant dont la chirurgie ambitieuse amène moins ses malheureux patients à un prompt rétablissement qu'à une dégradation certaine telle que la cécité ou l'impuissance. Lors d'une scène loufoque qui va précipiter la mort du poète, il opère un Rubén Darío terrifié pour lui extraire du foie un pus inexistant.

Or, il se trouve que d'une part, Debayle est «yankista» (246), et qu'il devient - bien malgré lui, il est vrai - le beau-père du tyran (sa fille Salvadora ayant épousé Somoza), ce en quoi il est associé respectivement à la perte de souveraineté et à l'ignominie. D'autre part, Darío, cet enfant du pays qui accuse quant à lui l'expansionnisme des Nord-Américains rabaissés à de grands singes brutaux (souvenons-nous de «gorilas»), incrimine également, à plusieurs reprises, l'arrivisme et l'impéritie de Debayle qu'il taxe de «bárbaro» lors de sa propre opération jusqu'à s'écrier: ¡Me van a matar! comme si le docteur, par ailleurs comparé à un "carnicero"(244-246), n'était guère différent en définitive du brutal voisin du nord et du sanglant dictateur. On n'oubliera pas l'histoire invraisemblable, mais non moins véridique, de l'imposant cerveau de Darío, que tentent de s'arracher le beaufrère du poète et Debayle, jusqu'à ce que l'urne se brise et que des marines, alertés par les cris, récupèrent «that damned thing! » (277).

Ce Rubén Darío icône du Nicaragua mais dont le cerveau à terre est réduit à une masse spongieuse, ce grand poète méprisé, pour ne pas dire insulté, dont le corps finit quasiment mutilé par un Debayle indirectement relié à la dépossession et à la sauvagerie, ne figure-t-il pas l'identité nationale blessée? Sauvagerie, écrivons-nous car à ces exemples de mutilation s'ajoute, nous le savons, celui de Quirón, héritier en un sens de Darío et victime des mêmes bourreaux des corps, rendu muet par des marines et sans doute par les soins toujours funestes du redoutable docteur.

Si l'on considère que les personnages de ces romans expriment un au-delà d'eux-mêmes, à savoir qu'ils renvoient à la patrie et à des valeurs nationales, et si l'on n'omet pas que l'identité fait corps avec la corporalité elle-même ou tout au moins avec l'image du corps, chacune façonnant pour partie l'autre, on perçoit ici la représentation douloureuse et dramatique du Nicaragua que dévoilent, par-delà les mots, les corps malmenés de Sergio Ramírez. 


\section{Le corps mort de la patrie: entre corruption et illusions perdues}

Darío et Sandino éteints, Quirón sans voix, Rigoberto López Pérez sans vie et atrocement meurtri dans sa virilité, autant dire la parole et la vaillance arrachées violemment du corps, c'est un pays orphelin et inhumant ses mythes qui se dégage d'entre les lignes, un Nicaragua rudoyé et tronqué, ayant donc perdu son intégrité, et qui ne se fait plus entendre - qui n'en a plus le courage? - condamné à l'oubli ainsi que nous le montrions il y a peu. Non moins qu'à la souillure, allégorique ici puisque traduisant de toute évidence la corruption de son propre dirigeant, père de la nation a-ton coutume de dire, qui paraît pourtant annihiler celle-ci en la vouant à la damnation.

Ce Nicaragua maculé et moribond est imagé de la façon la plus saisissante et épouvantable qui soit, par le biais d'un corps visible, dans Mil y una muertes où une photographie insoutenable clôt le roman. Elle illustre le rêve cataclysmique de l'un des deux narrateurs, un photographe du XIX $\mathrm{X}^{\mathrm{e}}$ siècle dénommé Castellón, visualisant la destruction du Nicaragua: "Sobre el gris sucio del barro que definía todo el horizonte, estaba tendido el cadáver desnudo de un niño de unos tres años. A su derecha, un cerdo negro y flaco lo husmeaba, acercándose» (Ramírez 2004: 351). Toute photographie est en elle-même considérée comme un témoignage du réel qu'elle authentifie par conséquent. De fait, la photo effroyable dont il est question ici, montre bien un fait avéré comme s'en explique Sergio Ramírez lui-même sur son site officiel, puisqu'elle fut prise:

en las vecindades del volcán Casitas, en el occidente de Nicaragua, después de que el huracán Mitch devastó al país en 1999. En el mar de lodo que quedó después del alud que bajó del volcán, el cadáver de un niño desnudo es acechado por un cerdo.

Cette vision terrible, si elle révèle un instant réel et veut désigner l'injustice de ces pays dits du tiers-monde où la misère entraîne une déchéance de l'être humain, est, à notre sens, lourde de symboles: il se pourrait même qu'elle recèle à elle seule les obsessions de l'auteur. Nous avons déjà pu apprécier le réseau de métaphores obsédantes qui imprègne les trames romanesques de Sergio Ramírez, et il nous semble que cette représentation du corps mort d'un enfant gisant dans la boue cristallise les démons de l'écrivain. En quoi le sens de cette image déborde-t-il cette dernière en signifiant plus que ce qu'elle donne à voir? Plusieurs éléments retiendront notre attention du fait de leur récurrence dans l'œuvre de Ramírez: l'atmosphère de corruption suggérée 
par la bourbe et le porc, puis la représentation de la mort, notamment celle d'un enfant au cœur d'un rêve.

Concernant le premier point, nous nous demandons si la fange de la photo ne s'inscrit pas dans le prolongement du thème excrémentiel étudié auparavant, au sein d'une sorte d'esthétique de la corruption, qui sert les desseins accusateurs du romancier. Dans Un sandinismo en el que creer, l'écrivain dénonce ce fléau persistant: "Vivo en una Nicaragua ahora envilecida por la corrupción y por las componendas políticas, que sufre bajo el peso de la marginación y la miseria, y donde no parecerían quedar vestigios de lo que fue un día la hermosa gesta revolucionaria que sacudió el continente» (Ramírez 2000: 29).

À la lumière de ce jugement, ce n'est sans doute pas un hasard si le porc est indirectement associé à l'Histoire du Nicaragua dans deux romans: dans Margarita, está linda la mar, Rubén Darío évoque le premier gouverneur du pays, Pedrarias Dávila, en ces termes : «Un conquistador que se hizo poderoso criando chanchos. Éste es un país bueno para engordar chanchos» (68). Un premier gouverneur aussi sanguinaire que celui qui, au $\mathrm{XX}^{\mathrm{e}}$ siècle, répandra la peur; un Somoza aussi engraissé par ses malversations que l'animal répugnant auquel il paraît implicitement relié: la deuxième partie de la citation empruntée au poète contient une ironie à peine voilée; surtout lorsqu'on sait qu'au Nicaragua le Palais National où siège le législateur est appelé «La Chanchera».

Dans Mil y una muertes ce pourceau qui métaphorise l'exercice corrompu du pouvoir, est à nouveau rattaché aux origines du Nicaragua post-colombien puisque son Histoire n'émanerait pas d'un savant discours entre le cacique Nicarao et le conquérant Gil González Dávila comme le prétendent les historiographes, mais d'une querelle, à bord du bateau du Découvreur: «esa historia se inició con la pelea entre un extraño animal mutilado [...] y un puerco de monte enloquecido de terror" (61). C'est donc encore la mutilation - et partant la perte d'intégrité? - et cet animal "impur" associé aux immondices et inspirant le dégoût qui définissent, depuis "son plus jeune âge", l'être même du Nicaragua si l'on ose dire. Ainsi est-il né, ainsi périt-il, du feu - purificateur? - d'un volcan aux résonances de «châtiment divin ", comme un écho d'un roman éponyme de 1988 écrit alors même que Sergio Ramírez assumait le pouvoir aux côtés de Daniel Ortega dont il fut, de 1984 à 1990, le vice-président.

D'autre part, sur la photo qui nous occupe, le porc apparaît dans tout un contexte nauséabond de mort. Cette thématique est déterminante dans 
les fictions de Sergio Ramírez où, indépendamment de sa présence souvent centrale, elle structure parfois le récit et oriente à elle seule la diégèse. Dans le roman, Castellón, photographe morbide et impudique, ne cache pas son attirance pour les clichés obscènes et sordides comme suffisent à en témoigner, outre les photos de nus, celle qu'il fait de sa propre fille tout juste assassinée sous ses yeux par les nazis, ou le cadavre nu de Tourguéniev pris à la dérobée, ou encore ces pendus dans un camp de concentration parmi lesquels des enfants.

Cette exhibition de la finitude du corps et de l'être, cette dramatisation de la corporéité au moyen d'un érotisme macabre, éveille chez le narrataire un malaise, peut-être même le malaise existentiel. Prétendant être en quête du mystère de l'âme, Castellón espère lire dans les corps nus ou morts, dépouillés de toute facticité, le secret de l'être; il aspire à voir l'invisible de chacun. Cette approche voyeuriste, et en un sens perverse, relie la vision du corps à une forme de violation et à la transgression. Sinon à la profanation, est-on tenté d'écrire, comme si Castellón attentait au "sacré" en l'homme? La photographie que reproduit Sergio Ramírez dans Mil y una muertes peut certes évoquer une désacralisation de la corporalité, mais ne montre-t-il pas l'horreur pour rappeler qu'il y a précisément des "choses" sacrées? Ou ne recourt-il pas à une image inconsciente du corps comme étant sacré pour mieux provoquer l'indignation et la compassion du lecteur?

Il nous reste d'ailleurs à explorer cette photo plurivoque qui clôt Mil y una muertes, dans son aspect le plus éprouvant pour le lecteur: le cadavre, seule "vision" de la mort pour le vivant, s'avère ici d'autant plus indécent et scandaleux, et renvoie d'autant plus au non-sens le non-être, qu'il s'agit de celui d'un enfant. Ce n'est pas le corps en lui-même qui est monstrueux en l'occurrence, mais sa représentation sépulcrale que la présence de l'enfant rend plus "irreprésentable" encore - comme une vision interdite? Nous nous interrogeons sur cette "mort de l'innocence" dans laquelle nous percevons une possible allégorie de l'Histoire du Nicaragua: ne peut-on interpréter ce rêve de mort comme la mort d'un rêve: le rêve sandiniste?

Expliquons-nous: il se trouve d'une part que Sergio Ramírez intitule le chapitre de son autobiographie consacré au triomphe de la Révolution: «La edad de la inocencia» (Ramírez 1999: 57) et innocents, ils l'étaient en un sens, ces sandinistes qui espéraient faire renaitre le pays; d'autre part, le rêve prophétique de Castellón le projette dans un Nicaragua des années 1990, et l'on sait que la débâcle sandiniste se produisit le 25 février 1990; un sandinisme qui, pour parler comme l'auteur, avait perdu ses valeurs éthiques, 
ce qui est selon lui le signe d'une mort prématurée (Ramírez 2000 : 31): nous sommes tentée de relier cette mort précoce due à la corruption (la perte de l'éthique), à l'enfant mort de la photo, cerné par l'impur.

Après la mort des mythes nationaux que suggère Margarita, está linda la mar, Mil y una muertes ne donne-t-il pas à lire la mort de cet autre mythe que fut la Révolution? Une mort dont Sergio Ramírez porte sans doute plus que d'autres la blessure au regard de son implication dans la victoire révolutionnaire et la reconstruction sociale. Il ne fait d'ailleurs aucun secret de sa désillusion, un thème récurrent dans ses divers écrits. Et pour cause, car celui qui intitule le chapitre de son autobiographie consacré cette fois à la révolution "trahie", "Las fauces de Saturno" - puisqu'elle ne tint pas ses promesses auprès des plus humbles et se trouva elle aussi salie par la corruption de certains - vit périr ses compagnons de lutte, assista à la déroute du sandinisme enfin sanctionné par le peuple las de la guerre civile, se désolidarisa de Daniel Ortega avec lequel la querelle se poursuit à en juger par le blog de l'auteur qui fait le procès de ses abus de pouvoir, créa en 1995 le Movimiento de Renovación Sandinista mais n'obtint à la présidentielle de 1996 qu'un pour cent de votes pour un million de dollars de dette.

La terrible photo de Mil y una muertes ne se fait-elle pas l'écho des illusions perdues de Sergio Ramírez? avec ce corps qu'on peut envisager comme une métaphore du petit pays expirant dans une atmosphère corrompue: souillé et oublié de tous. Un corps charnel pour dire l'identité d'un corps terrestre, tous deux meurtris; des corps de chair grotesques, monstrueux, mutilés, pour signifier cet au-delà d'eux-mêmes, leur patrie, dont l'Histoire s'avère tout aussi risible et cruelle si l'on en croit les propos de l'écrivain : «la historia de Nicaragua es una burla sangrienta» (Ramírez 1985: 303). D’où l'horreur et la dérision, d'ailleurs appliquées à toute chose, car cette farce sanglante, n'est-ce pas en dernière instance la vie même? avec, comme unique arme contre la mort, le rire.

L'image du corps, qui ressortit en partie à l'histoire individuelle, ainsi qu'à la société dont le corps va précisément "incorporer" les valeurs et la culture, paraît dépendre également chez Sergio Ramírez d'une Histoire collective, probablement intériorisée elle aussi. Comme la cicatrice d'un désenchantement en pleine chair.

Si la corporéité est le vecteur des symboles dont use une société, mais aussi l'écrivain, pour dire ses angoisses et ses désirs, on peut ici se demander si les nicas ressentent leur Histoire, ou appréhendent le corps de la nation, au moyen des mêmes images que celles des romans que nous venons 
de commenter. Les représentations fantasmatiques de Sergio Ramírez rendent-elles compte d'un imaginaire ou d'un inconscient nicaraguayen? Dévoilent-elles - ou contribuent-elles éventuellement à façonner - de nouveaux archétypes? Sergio Ramírez est-il cette voix du peuple qu'il a toujours souhaité refléter et, de manière plus générique, dans quelle mesure un écrivain fait-il corps avec les siens, avec sa terre? Dans quelle mesure incarne-t-il l'esprit du lieu?

Gilbert Durand estime qu'une grande œuvre littéraire est:

celle où l'on s'élève des images obsédantes et du «mythe personnel» chers à la psychocritique de Charles Mauron, jusqu'à ces mythes qui hantent une collectivité d'hommes tout entière à travers le temps d'une histoire culturelle ou dans les instants privilégiés qu'enchâssent les grands événements sociaux? (Durand 1992: 239)

De l'être-à-soi à l'être-au-monde, le roman qui "voit juste" serait celui qui, émanant d'une appréhension personnelle, ouvre sur une sorte de psyché - oserait-on écrire d'intimité? - collective; celui qui formule des sentiments ressentis dans les profondeurs du peuple, parce que l'écrivain n'est pas seulement quelqu'un qui écrit avec tout son corps et tout son être, mais également celui qui s'exprime pour ses compatriotes, et plus amplement pour ses semblables de quelque géographie qu'ils soient. Dans un insaisissable corps à corps?

\section{Bibliographie}

Bakhtine, Mikhaïl, 1985, L'œuvre de François Rabelais. (La culture populaire au Moyen-Âge et sous la Renaissance), Paris, Éditions Gallimard.

Bergson, Henri, 1993, Le rire, Paris, Collection Quadrige, PUF.

Durand, Gilbert, 1992, Figures mythiques et visages de l'œuvre. De la mythocritique à la mythanalyse, Paris, Éditions Dunod.

Durkheim, Émile, 1968, Les formes élémentaires de la vie religieuse, Paris, PUF.

Duval, Sophie, Martinez, Marc, 2000, La satire. (Littérature française et anglaise),

Paris, Collection U, Série Lettres, Armand Colin.

Freud, Sigmund, 1979, «Le Moi et le Ça», in Essais de psychanalyse, Traduction de S.

Jankélévitch, Paris, Petite Bibliothèque Payot.

Lascault, Gilbert, 2004, Le monstre dans l'art occidental, Paris, Éditions Klincksieck. Carol, Anne, 2005, in Régis Bertrand et Anne Carol (ss dir.), Le «monstre» humain.

Imaginaire et société, Publications de l'Université de Provence. Ramírez, Sergio, 1985, El alba de oro, México, Siglo XXI Editores.

Ramírez, Sergio, 1998, Margarita, está linda la mar, Madrid, Ediciones Alfaguara. 
Ramírez, Sergio, 1999, Adiós Muchachos. Memoria de la revolución sandinista, Madrid, México, El País/Aguilar.

Ramírez, Sergio, 2000, Un sandinismo en el que creer. In Oficios Compartidos, Poitiers, CRLA-Archivos, "Conferencias en el centro".

Ramírez, Sergio, 2002, Sombras nada más, México, Alfaguara.

Ramírez, Sergio, 2004, Mil y una muertes, México, Alfaguara.

Ramírez, Sergio, 2004, Una vida por la palabra. Entrevista de Silvia Cherem, México, Fondo de Cultura Económica.

Ramírez, Sergio, site officiel: http://www.sergioramirez.org.ni/

Ramírez, Sergio, blog El Boomeran(g): http://www.elboomeran.com/blog/7/sergioramirez/ 